\title{
Development of Highly Sensitive Ru-Chelate Based ECL Immunoassay 2: Electrochemical and Immunochemical Studies on Homogeneous and Heterogeneous ECL Excitation
}

\author{
Yuzaburo NAmBa,* Takashi Sawada,* and Osamu SuzukI** \\ *Clinical Diagnostics Department, Eisai Co., Ltd., 5-5-5 Koishikawa, Bunkyou, Tokyo 112-8088, Japan \\ **Graduate School of Advanced Sciences of Matter, Department of Molecular Biotechnology, \\ Hiroshima University, 1-4-1 Kagamiyama, Higashi-hiroshima, Hiroshima 724-8527, Japan
}

\begin{abstract}
Studies on the electrochemical and immunochemical reaction kinetics of the heterogeneous type of ECL excitations were made comparatively with homogeneous types of ECL excitations by measuring human IgG (hIgG) using an antibody labeled with a ruthenium(II) tris(bipyridyl) (Ru-chelate) as the luminophore (Ru-Ab). Solid-phase sandwich-type immunoassays were carried out on the surface of magnetic micro-beads (MB) with a diameter of $4.5 \mu \mathrm{m}$. In the ECL measurement, two types of ECL excitation methods were compared. One was a homogeneous ECL excitation, where the reacted $\mathrm{MB}$ together with non-reacted $\mathrm{Ru}-\mathrm{Ab}$ were excited in a suspending phase without any bind/free (B/F) separation procedure. The other was a heterogeneous one where the reacted MB were excited over the electrode after being collected by a magnet following the $\mathrm{B} / \mathrm{F}$ separation to remove the non-reacted Ru-Ab. In electrochemical studies, it was revealed that the $\mathrm{Ru}-\mathrm{Ab}$ reacted with $\mathrm{hIgG}$ decreased the ECL emission efficiency. The decreasing ratio was inversely correlated with the cubic root of the luminophore molecular weight. In homogenous ECL excitation for the reacted matrix containing both the reacted $\mathrm{MB}$ and the non-reacted $\mathrm{Ab}$, however, a reverse correlating dose response curve appeared only in the area beyond the antigen-antibody equivalent point, the so-called antigen excess zone; as a result the $\mathrm{S} / \mathrm{N}$ ratio of the ECL signal was as small as only 1.3. In contrast, the heterogeneous ECL excitation for the reacted MB, with the non-reacted $\mathrm{Ru}-\mathrm{Ab}$ removed by $\mathrm{B} / \mathrm{F}$ separation, demonstrated 1000 or more times the $\mathrm{S} / \mathrm{N}$ ratio in the area before the antigen-antibody equivalent point. Thus, this heterogeneous ECL excitation with B/F separation improved the detection sensitivity dramatically up to 1000 or more times higher than that of the homogeneous ECL excitation. Consequently, the sensitivity of heterogeneous ECLIA could be competitive with that of the conventional chemiluminescence immunoassay.
\end{abstract}

(Received March 7, 2000; Accepted April 21, 2000)

\section{Introduction}

Recently, several kinds of chemiluminescence (CL) method have been used for practical immunoassay, such as chemiluminescence immunoassay (CLIA), chemiluminescence enzyme immunoassay (CLEIA) and electrochemiluminescence immunoassay (ECLIA), as a non-isotopic immunoassay. ${ }^{1-4}$ The former two luminescence immunoassay systems (CLIA and CLEIA) employed an oxidization procedure for the luminophore (acridinium ester or luminol) by hydrogen peroxide that was added, or produced by the labeled enzyme, or employed another emission-producing method through dephosphorylation for the luminophore (1,2-dioxethane compound: AMPPD). Consequently, in order to maintain good reproducibility, these luminescence reactions require operation under a highly automated procedure to eliminate the influences of several factors, such as temperature and the timing of adding and/or mixing reagents, etc. On the other hand, the ECL emission was produced through oxidization of the luminophore

$\doteqdot$ To whom correspondence should be addressed. by an electrochemical oxidation procedure. ${ }^{5}$ In the ECL system, since not only the initiation, but also the entire process of the luminescence, can be controlled by the electric potential of the electrode, the reproducibility of the ECL can be more easily secured compared with the CL methods. While the ECL emission provides these beneficial features, an electrochemical oxidative and/or reductive reaction occurs within a limited reaction layer only, the so-called electrical double layer and/or the electrical diffused layer, at several $\mathrm{nm}$ distances from the surface of the working electrode (WE). ${ }^{6}$ For this reason, the ECL phenomenon occurs only within a very limited area of the solution close to the electrode surface, not in an entire area of the solution, such as in other luminescence systems. Consequently, no satisfactory result has been obtained by such an ECLIA method of simply replacing the conventional labeled compound with the ECL luminophore. For example, in the initial ECLIA reported by Ikariyama, which used human serum albumin (HSA) labeled with pyren as the luminophore, the detection sensitivity was as low as the $\mu \mathrm{mol} / \mathrm{ml}$ level, and the measurement range was very narrow (i.e. $\mathrm{S} / \mathrm{N} \leqslant 2.5$ ). ${ }^{7}$ Similar results with antigen-labeled competitive ECLIA were reported by Massey and Shah using a ruthenium(II) tris(bipyridyl) (Ru- 
chelate) based ECL, of which emission efficiency was extremely enhanced by the simultaneous electrolysis of TPA in the electrolytes solution, measuring the immunoglobulin or digoxin. ${ }^{8-10}$ These ECLIA have an advantage of performing the homogeneous assay without any bind/free (B/F) separation throughout the entire procedure from immunoassay to ECL excitation. These homogeneous ECLIA methods, however, tended to measure the ECL emissions produced by the nonreacted luminophore, because the ECL signals were correlated with the concentration of the analyte antigen that was not labeled by luminophore. Consequently, these homogeneous ECLIA provided only low $\mathrm{S} / \mathrm{N}$ ratios and narrow measurement ranges, showing the high background ECL emission.

In a preceding study, using both the Ru-chelate and TPA, the same ECL system as that of Massey and Shah, the authors reported a new heterogeneous ECLIA method, which could provide high sensitivity at the picogram level and also a remarkably wide detecting range of 1000 to 10000 fold magnitude levels. ${ }^{11}$ In this method, the sandwich immunoassay was carried out using paramagnetic micro-beads (MB) as a solid-phase carrier. The antibody labeled with $\mathrm{Ru}$-chelate $(\mathrm{Ru}-$ $\mathrm{Ab}$ ) reacted with an antigen which was bound on the surface of the $\mathrm{MB}$, and the ECL excitation with the reacted MB was performed, collecting it over the electrode surface by a magnet after B/F separation. Since this heterogeneous ECL excitation could eliminate any possible ECL emission from the nonreacted luminophore, the background emission was tremendously reduced, and such a high $\mathrm{S} / \mathrm{N}$ ratio as several ten thousand fold levels were obtained.

This report deals with comparative studies on the reaction kinetics between the obsolete homogeneous ECLIA and the newly developed heterogeneous ECLIA. In these studies, we found the phenomenon that the ECL emission efficiency was reduced due to an increase in the molecular size of the luminophore complex with the antigen-antibody reaction in the homogeneous ECL excitation. We also found that the actual dose-response curve decreasing in the ECL intensity was obtained only in the area beyond the antigen-antibody equivalent point, the so-called antigen excess zone, where a greatly excessive amount of the antigen reacted with the antibody. On the other hand, the heterogeneous ECLIA made it possible to directly measure the reacted immune complex conformed in front of the antigen-antibody equivalent point, which has already been utilized for the majority of the current highly sensitive immunoassay systems. Consequently, it was revealed that theoretically the sensitivity of the heterogeneous ECLIA was 1000 times or more superior to that of homogeneous ECLIA.

These experimental studies and the results thereof are given below.

\section{Experimental}

Electrolyte solution (ES) and flow-cell cleaner

The composition of the electrolyte solution (ES) was the same as that previously reported. ${ }^{11}$

Ru-chelate solution

A $37.6 \mathrm{mg}$ amount of tris(bipyridyl)ruthenium(II)chloride (Ru-chelate; Aldlich Chemical Co., Milwaukee, USA) was dissolved into $50 \mathrm{ml}$ of ES to make a $1 \mathrm{mM} \mathrm{Ru}$-chelate solution, and further diluted with ES by a serial ten-fold dilution up to a level of $0.1 \mathrm{pM}$ of Ru-chelate solution.

\section{$M B$ coated with antibody (Capt-MB)}

The monoclonal anti-human $\operatorname{IgG}(\mathrm{Fd})$ antibody (Wako Pure Chem. Co. Ltd., Tokyo, Japan) was used as the capture antibody (Capt-Ab) for the sandwich immunoassay. As a solid-phase carrier for the Capt-Ab, paramagnetic micro-beads (MB), each with a diameter of $4.5 \mu \mathrm{m}$ (Dynabead ${ }^{\circledR} \mathrm{M}-450$, Dynal, Oslo, Norway), were used. The Capt-Ab was coated over the surface of the MB by a physical adsorptive method, according to a previously reported procedure. ${ }^{11}$

\section{Antibody labeled with Ru-chelate (Ru-Ab)}

As the 2nd antibody (2nd-Ab), the monoclonal anti-human $\mathrm{IgG}(\mathrm{Fc})$ antibody (Wako Pure Chem. Co. Ltd., Japan) was used. Labeling of the $\mathrm{Ru}$-chelate to the $2 \mathrm{nd}-\mathrm{Ab}$ was carried out according to a previously reported procedure. ${ }^{11}$ The labeled antibody in the protein concentration of $148 \mu \mathrm{g} / \mathrm{ml}$ was diluted to 500, 1500 and 3000 fold with PBS (pH 7.5) containing $10 \%$ of normal rabbit serum (NRS) and $0.02 \%$ of $\mathrm{NaN}_{3}$ before use as the antibody labeled with Ru-chelate-NHS (Ru-Ab).

\section{$M B$ coated with Ru-Ab (Hot-MB)}

As a material to investigate the heterogeneous ECL excitation without any immunoassay procedures, MB coated with $\mathrm{Ru}-\mathrm{Ab}$, Hot-MB was prepared according to a previously reported procedure. ${ }^{11}$

\section{ECL detection apparatus and methods}

Although the ECL detection apparatus used was the same as that in a previous report, ${ }^{11}$ the electricity was slightly changed to improve both the low- and high-end resolutions of the photon detection. A photon multiplier tube (PMT) module unit H678001 (Hamamatsu Co., Hamamatsu, Japan), which has a widerange linearity of photon current, more than $10^{6}$, was used. The photon-signal current detected by the PMT was processed by a personal computer after being digitized by a high-speed $(10 \mu \mathrm{s})$ digital to analog converter (A/D converter) in the ratio of $69 \mathrm{pA}$ to one digit. The mean value of 1000 photon-signals for a 10 ms period was used as an arbitrary unit (a.u.) of ECL intensity. For ECL excitation, the sweep electric potential or the constant electric potential was applied upon the electrode. For measuring the ECL emission of a specimen containing the reacted $\mathrm{MB}$ with non-reacted $\mathrm{Ru}-\mathrm{Ab}$ or without non-reacted $\mathrm{Ru}-$ $\mathrm{Ab}$ by $\mathrm{B} / \mathrm{F}$ separation, the following three different $\mathrm{ECL}$ excitation procedures were performed. (1) Liquid-phase excitation (LP-exc): The specimen without B/F separation was filled into the flow cell electrode, followed by the ECL excitation with the reacted MB in suspension; (2) Just magnet sediment excitation (JM-exc): The specimen without $\mathrm{B} / \mathrm{F}$ separation was filled into the flow cell electrode, followed by sedimentation of the reacted MB upon the surface of the WE by a momentary contact of a magnet with the backside of the electrode, and then applying the ECL excitation; (3) Magnetic trapping excitation (MT-exc): As the reacted MB with $\mathrm{B} / \mathrm{F}$ separation passed completely through the flow cell electrode, a magnet was contacted with the backside of the WE in order to collect all the MB upon the surface of the WE, followed by applying the ECL excitation, as shown in Fig. 1.

After ECL excitation, flow cell cleaning solution with a high $\mathrm{pH}$ was drawn into the flow cell chamber, and the electrochemical cleaning procedure was performed in order to restore and maintain the reproducibility of the ECL excitation.

\section{Specimen}

For the antigen of the immunoassays, normal human serum containing $12 \mathrm{mg} / \mathrm{ml}$ of hIgG was used at a concentration of 30 


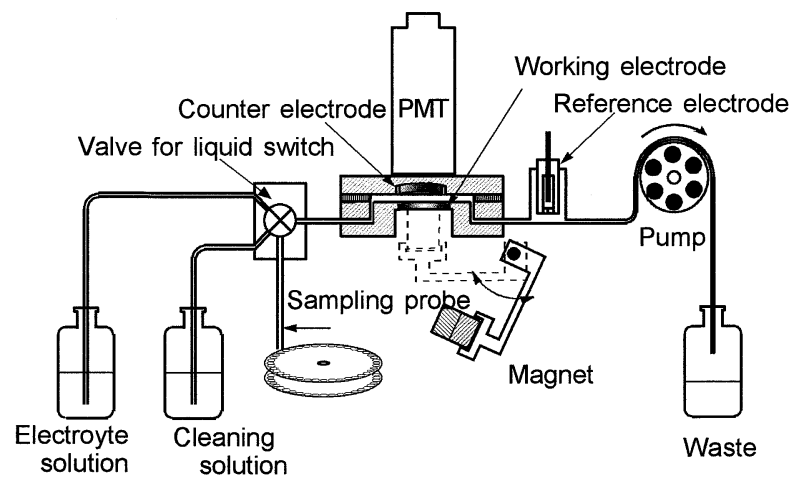

Fig. 1 Schematic composition of ECL detection system. For the ECL measurement, 3 types of excitation method, (LP-exc, JM-exc and MT-exc) were operated. In LP-exc, the sample was simply filled into a flow cell and ECL excitation was made. In JM-exc, after filling the sample the magnet arm was moved upward for a moment, causing sedimentation of the MB on the WE followed by ECL excitation. In MT-exc, the magnet arm was positioned upward during the measurement specimen with $\mathrm{B} / \mathrm{F}$ separation passing through the cell to collect all the MB on the WE.
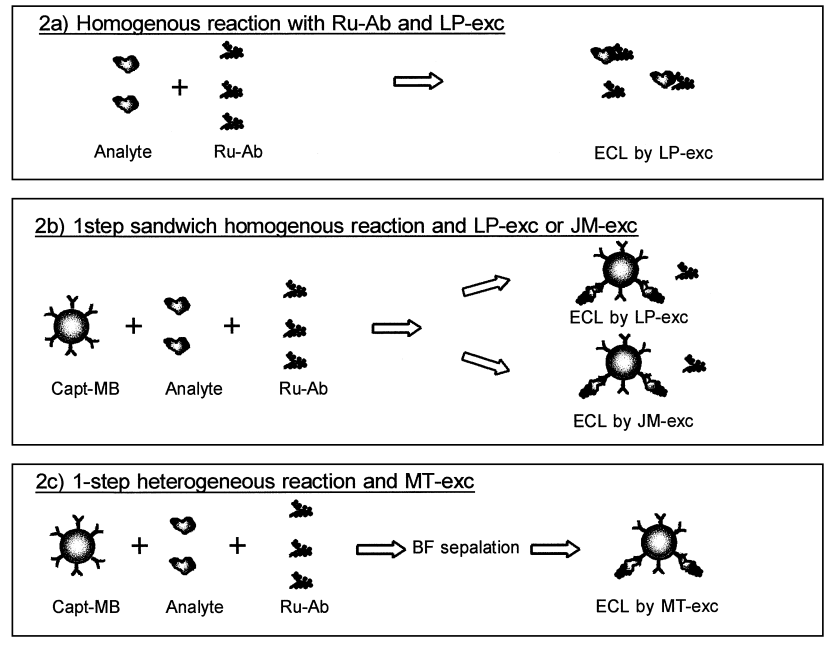

2d) 2-step heterogeneous reaction and MT-exc

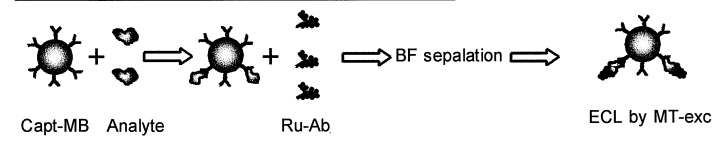

Fig. 2 Schematic illustrations of the immunoassay methods and ECL excitation procedures. 2a) Homogeneous reaction of the hIgG with $\mathrm{Ru}-\mathrm{Ab}$ and detection by LP-exc. 2b) The 1-step sandwich homogeneous reaction with an analyte and the detection by LP-exc or JM-exc. 2c) The 1-step heterogeneous reaction with analyte and the detection by MT-exc. 2d) The 2-step heterogeneous reaction with analyte and the detection by MT-exc.

$\mu \mathrm{g} / \mathrm{ml}$ to $1 \mathrm{ng} / \mathrm{ml}$ by serial dilution with $0.1 \mathrm{M}$ PBS ( $\mathrm{pH} 7.5$ ) containing $0.02 \%$ of $\mathrm{NaN}_{3}$ and $10 \%$ of NRS.

\section{Immunoassay}

Figures $2 \mathrm{a}, 2 \mathrm{~b}, 2 \mathrm{c}$ and $2 \mathrm{~d}$ schematically illustrate 4 types of ECLIA carried out for these studies: 1) Homogeneous reaction and LP-exc (Fig. 2a); a $20 \mu \mathrm{l} \mathrm{hIgG} \mathrm{standard} \mathrm{specimen} \mathrm{was}$ added to $100 \mu \mathrm{Ru}-\mathrm{Ab}$ in a reaction tube, and was incubated for $2 \mathrm{~h}$ at RT with mixing. Then, $500 \mu \mathrm{l}$ of ES was added into the

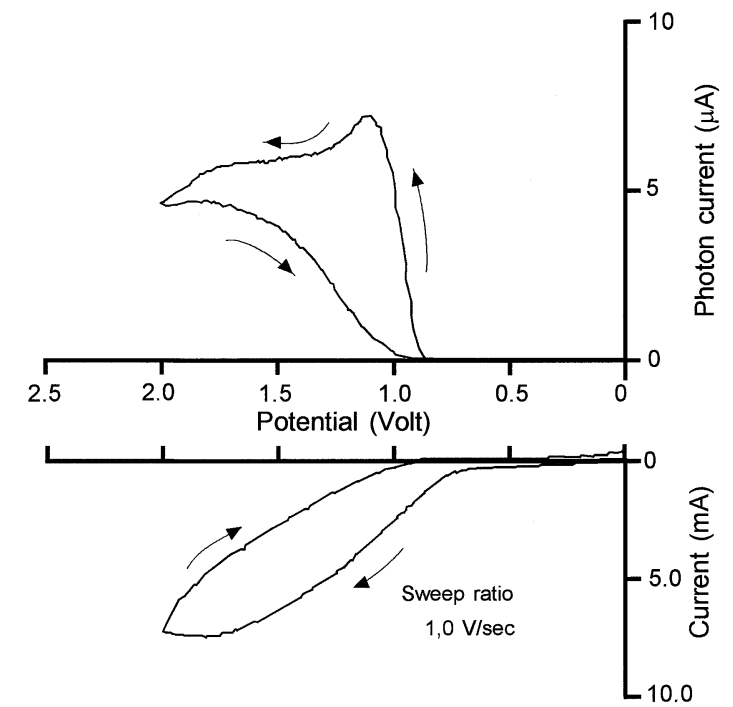

Fig. 3 Cyclic voltammogram and ECL emission of a $10 \mathrm{nM} \mathrm{Ru}-$ chelate solution.

reaction tube for ECL measurement and was made LP-exc. 2) 1 -step sandwich homogeneous reaction and LP-exc or JM-exc (Fig. 2b); all of $50 \mu \mathrm{l}$ Capt-MB as well as $20 \mu \mathrm{l}$ of hIgG standard specimen and $100 \mu \mathrm{l} \mathrm{Ru}-\mathrm{Ab}$ were reacted at the same time in a reaction tube for $2 \mathrm{~h}$ with mixing at RT. Then, $500 \mu \mathrm{l}$ of ES was added into the reaction tube. The ECL measurement of the reacted solution was made by LP-exc and JM-exc. 3) The 1-step heterogeneous reaction and MT-exc (Fig. 2c); all of $50 \mu \mathrm{l} \mathrm{Capt-MB}$ as well as $20 \mu \mathrm{l}$ of hIgG standard specimen and $100 \mu \mathrm{Ru}-\mathrm{Ab}$ were reacted at the same time for $2 \mathrm{~h}$ with mixing at RT. Then, to remove the non-reacted Ru-Ab, B/F separation was carried out and the washed MB was re-suspended in $300 \mu \mathrm{l}$ ES. A $250 \mu 1$ suspension of the MB was subjected to the ECL measurement by MT-exc. 4) The 2-step heterogeneous reaction and MT-exc (Fig. 2d); a $20 \mu \mathrm{l} \mathrm{hIgG} \mathrm{standard} \mathrm{specimen} \mathrm{and} 50$ $\mu 1$ Capt-MB were added into $100 \mu 10.15$ M PBS (pH 7.5) containing $10 \%$ of NRS, in a reaction tube and were incubated for $1 \mathrm{~h}$ at RT with mixing. Thereafter, the reaction medium was removed from the reaction tube by $\mathrm{B} / \mathrm{F}$ separation. A $100 \mu \mathrm{l}$ of $\mathrm{Ru}-\mathrm{Ab}$ was added into the reaction tube and incubated for $1 \mathrm{~h}$. Thereafter, the non-reacted $\mathrm{Ru}-\mathrm{Ab}$ was removed from the reaction tube by $\mathrm{B} / \mathrm{F}$ separation, and the reacted $\mathrm{MB}$ were resuspended in $300 \mu \mathrm{l} \mathrm{ES}$. A $250 \mu \mathrm{l}$ suspension of the MB was subjected to the ECL measurement by MT-exc.

\section{Results and Discussion}

ECL time course by voltage scans excitation

Figure 3 shows the results of a cyclic voltammogram of the $100 \mathrm{nM}$ Ru-chelate solution by a scanning rate of $1.0 \mathrm{~V} / \mathrm{s}$. The current started to flow after the electric potential exceeding 0.75 $\mathrm{V}$, and the peak current appeared at an electric potential of around $1.7 \mathrm{~V}$. Meanwhile, the ECL emission appeared at the electric potential of $0.85 \mathrm{~V}$ to $0.9 \mathrm{~V}$, and steep peak emission was observed at an electric potential of $1.0 \mathrm{~V}$; thereafter, the emission continued during the period that the oxidative current was flowing, although the emission intensity became weaker gradually.

Figures $4 \mathrm{a}$ and $4 \mathrm{~b}$ show the ECL emission time courses of a Ru-chelate solution $($ M.W. = 750), Ru-Ab (M.W. = 150000), 


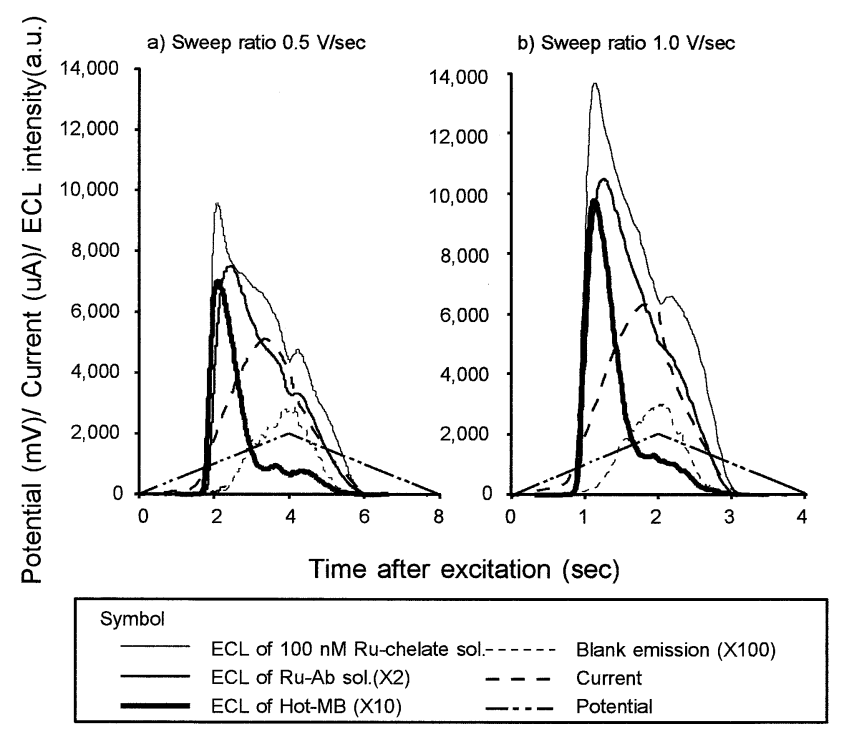

Fig. 4 ECL time courses for different molecular sizes of luminophore, Ru-chelate solution (M.W. = 750), Ru-Ab (M.W.= 150000 ), and Hot-MB (the diameter $=4.5 \mu \mathrm{m}$ ) by voltage scanning excitation.

and Hot-MB (the diameter $=4.5 \mu \mathrm{m}$ ) excited by the scan rates of $0.5 \mathrm{~V} / \mathrm{s}$ and $1.0 \mathrm{~V} / \mathrm{s}$, respectively. The MT-exc was given to the luminophore in a suspension form, Hot-MB, and the LP-exc were given to the luminophore in liquid forms, Ru-chelate and $\mathrm{Ru}-\mathrm{Ab}$. Since the magnitude of the ECL intensities varied with each specimen, the ECL values shown in Figs. $4 \mathrm{a}$ and $4 \mathrm{~b}$ are indicated as enlargements of the original ECL intensity by 2 times for the Ru-Ab, 10 times for the Hot-MB, and 100 times for the blank emission.

Higher ECL emissions were observed at a higher scanning rate, $1.0 \mathrm{~V} / \mathrm{s}$ (Fig. 4b). The potential at the peak ECL emission was around $1.15 \mathrm{~V}$ for the Ru-chelate and the Hot-MB, and 1.25 to $1.3 \mathrm{~V}$ for the $\mathrm{Ru}-\mathrm{Ab}$, regardless of the scanning rate. The potential at the peak ECL emission for the Ru-Ab specimen was slightly higher than that for the others, which might be caused by a change in the composition of the ES by adding $10 \%(\mathrm{v} / \mathrm{v})$ of PBS dissolving the Ru-Ab. The ECL emission curve of the Hot-MB with the huge molecular size of $4.5 \mu \mathrm{m}$ was mainly composed of the steep main peak. On the other hand, the ECL emission curve of the Ru-Ab (M.W. of 150000) and that of Ruchelate (M.W. of 750) included the main peaks as well as the second and third peaks, respectively. These second peaks appeared at a potential of $1.6 \mathrm{~V}$ to $1.7 \mathrm{~V}$, where the current was the maximum; the third peaks appeared after the maximum potential, where the blank emission reached the maximum levels, as shown in Figs. $4 a$ and $4 b$.

These side peaks, which appeared at the electric potential exceeding the water hydrolysis potential, became higher as the molecular size of the luminophore complex became smaller; in other words, as it moved more easily. In addition, these side peaks became higher with increasing scanning ratio of the potential. The reason for these side peaks appearing for the small size of luminophore was considered to be due to the deflagrated molecular motion by excess electrolysis, and that not only the TPA, but also the small-sized luminophore, might be more oxidized by migration to WE located far from the double diffusion area such as the offshore zone.

Consequently, the voltage-scanning method was not an adequate excitation method, since the waveforms of the ECL
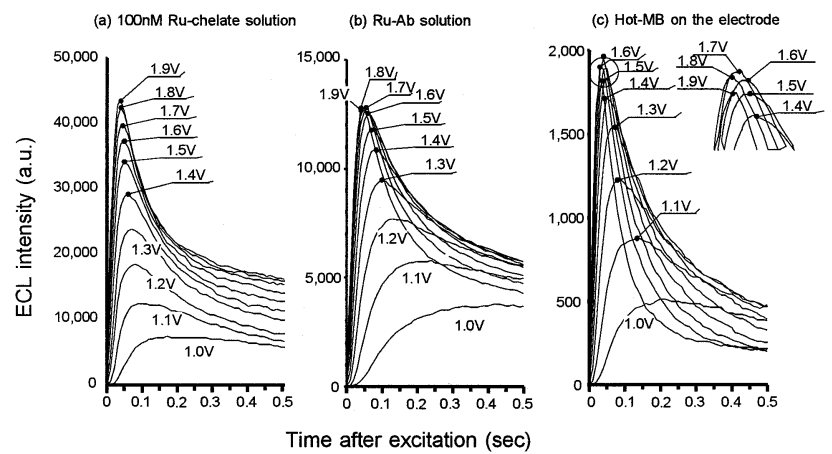

Fig. 5 ECL time courses for different molecular sizes of luminophore, (a) Ru-chelate solution (M.W. = 750), (b) Ru-Ab $($ M.W. $=150000)$, and $(\mathrm{c})$ Hot-MB (the diameter $=4.5 \mu \mathrm{m})$ by excited constant voltage at $1.4 \mathrm{~V}$.

emission varied due to the molecular sizes of the luminophore complex.

\section{ECL time course by constant voltage excitation}

Figures $5 \mathrm{a}, 5 \mathrm{~b}$ and $5 \mathrm{c}$ show the ECL emission time courses for the 3 different molecular sizes of the luminophore by exciting with constant voltage. In the case of the ECL excitation potential at $1 \mathrm{~V}$, the plateau ECL emissions were observed during a 200 to $400 \mathrm{~ms}$ period for every molecular size of the luminophores. As the potential was elevated, the steep peak of the ECL emission appeared more quickly, and at a potential exceeding $1.4 \mathrm{~V}$, the peak ECL emission appeared constantly within 40 to $50 \mathrm{~ms}$.

As shown in Fig. 5a, the ECL emission-peak value for the smallest molecular size luminophore, Ru-chelate, increased according to elevation of the potential. On the other hand, as shown in Fig. 5b, the ECL emission-peak value for the medium molecular-size luminophore, Ru-Ab, decreased when the potential exceeded 1.7 V. Likewise, as shown in Fig. 5c, the largest molecular size luminophore, Hot-MB $(4.5 \mu \mathrm{m}$ in diameter), decreased when the potential exceeded $1.6 \mathrm{~V}$. It was further observed that the decrease of the ECL emission after the peak became faster with an increase in both the molecular size and the potential. In contrast, as shown in Fig. 5a, the ECL emission intensity of the Ru-chelate, which was the smallest molecular size (750) luminophore among them, is indicated as always being higher at any higher excitation potential compared with at the lower excitation potential, even $0.5 \mathrm{~s}$ after excitation.

In ECL emission, the hook phenomenon which appeared for the larger molecular size luminophore began at a lower excitation potential when the TPA concentration decreased to $1 / 4$ of the initial concentration (not shown in the data). This hook phenomenon seems to occur when a larger size luminophore to be reduced lacks the co-operation of its partner, TPA radical, although a sufficient amount of TPA was contained in the ES. While the TPA located near the WE is rapidly oxidized by higher potential, but the re-oxidation of the luminophore in larger molecular size is still in slow turnover, and as a result of difference in the life time with each other the ECL emission of the larger molecules may be decreased. For this reason, the present experimental studies were made by the ECL excitation under a constant potential of $1.4 \mathrm{~V}$.

\section{ECL linearity responses of Ru-chelate solution}

Figure 6 shows the dose-responses of the ECL linearity of the $\mathrm{Ru}$-chelate solutions with concentrations of $1 \mathrm{pM}$ to $1 \mu \mathrm{M}$, at 


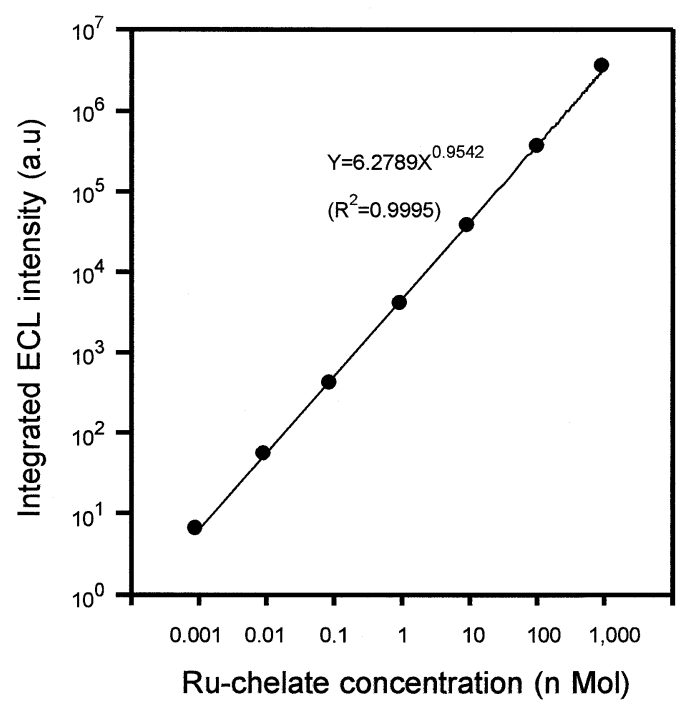

Fig. 6 ECL emission linearity of the Ru-chelate solution.

1.4 V excitation. The ECL intensity accumulated $200 \mathrm{~ms}$ of the signals during the period from $30 \mathrm{~ms}$ to $230 \mathrm{~ms}$ after the start of excitation. The linear ECL emission continued over the $10^{6}$ fold range of the Ru-chelate concentrations from pM levels up to high levels of $1 \mu \mathrm{M}$, at which the PMT outputs were saturated. The linearity of chemiluminescence emission, such as the luminol emission, whose intensity is varied by both concentrations of luminol and hydrogen peroxide, is limited to about $10^{3}$ to $10^{4}$.

Accordingly, the linearity range of $10^{6}$ in the ECL of Ruchelate could be emphasized as an exceedingly wide range. This wide range of ECL emission, which seemed to be correlated with the Ru-chelate concentration, could only be achieved by a reaction with a huge excess of TPA at $2 \times 10^{5}$ or more times the Ru-chelate. In a previous study by Blackburn, in order to measure the ECL emission at such a wide range as $10^{6}$ or more the PMT gain had been changed several times. ${ }^{4} \mathrm{We}$ were successfully able to measure this wide range of ECL emission without changing the PMT gain by using an improved PMT and using a mean value of 1000 of digital signals converted by a high speed A/D converter.

\section{Homogeneous ECLIA between IgG and Ru-Ab}

In the homogeneous ECLIA between the $\mathrm{hIgG}$ and the $\mathrm{Ru}-\mathrm{Ab}$, about 5\% decrease in ECL signals were observed in the reaction using the highly concentrated $\mathrm{hIgG}(30 \mu \mathrm{g} / \mathrm{ml})$ and the most diluted Ru-Ab (5 ng; 3000 fold diluted solution) by LP-exc. However, the degrees of ECL signal changes were not enough to deduce any dose-response curve between the ECL signals and the hIgG concentrations. Then, the ECL emission efficiency of the $\mathrm{Ru}-\mathrm{Ab}$, which completely reacted with the antigen, was compared with that of the non-reacted $\mathrm{Ru}-\mathrm{Ab}$. A $1 \mathrm{ml}$ antigen solution containing $300 \mu \mathrm{g} \mathrm{hIgG}$ and a $200 \mu \mathrm{l}$ solution containing $15 \mu \mathrm{g} \mathrm{Ru}-\mathrm{Ab}$ were reacted overnight. The reacted specimen was diluted with the ES 10 fold, followed by LP-exc. As shown in Table 1, the ECL emission efficiency of the molecular size in double, Ru-Ab reacted with hIgG (hIgG-Ru$\mathrm{Ab}$ ), was reduced to $81 \%$ compared with that of the non-reacted $\mathrm{Ru}-\mathrm{Ab}$.

On the basis of taking the $100 \mathrm{nM}$ Ru-chelate specimen as $100 \%$, the ECL intensity of the Ru-Ab per Ru-chelate mol concentration was $17.3 \%$, and that for the hIgG-Ru-Ab $14.1 \%$.
Table 1 ECL emission efficiency for the difference molecular size of luminophore

\begin{tabular}{|c|c|c|c|c|c|}
\hline Sample & ECL int. & $\begin{array}{c}\text { Ru-chelate } \\
\text { content/ } \\
\mathrm{nM}^{\mathrm{a}}\end{array}$ & $\begin{array}{c}\text { \% ECL int./ } \\
\text { nM } \\
\text { Ru-chelate }\end{array}$ & M.W. & $\begin{array}{l}\text { Cubic } \\
\text { root of } \\
\text { M.W. }\end{array}$ \\
\hline Ru-chelate & 416973 & 100 & 100.0 & 750 & 9 \\
\hline $\mathrm{Ru}-\mathrm{Ab}$ & 144412 & 200 & 17.3 & 150000 & 53 \\
\hline hIgG-Ru-Ab ${ }^{b}$ & 111777 & 200 & 14.1 & 300000 & 67 \\
\hline
\end{tabular}

a. Each absorptive value at $455 \mathrm{~nm}$ was divided by 13700 .

b. A $15 \mu \mathrm{g}$ of Ru-Ab in $200 \mu \mathrm{l}$ was reacted for overnight with a $300 \mu \mathrm{g}$ of hIgG in $1 \mathrm{ml}$ solution and diluted with the ES by 10 fold for ECL measurement.

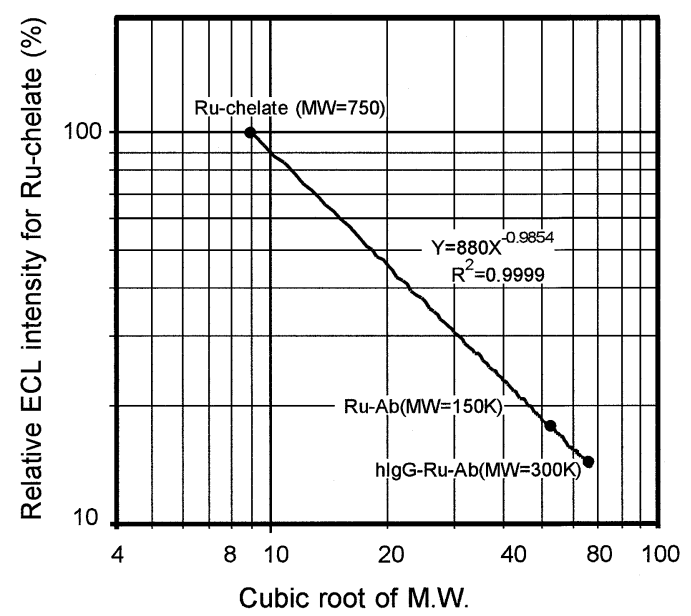

Fig. 7 ECL emission efficiency by molecular weight (M.W.) of the luminophore.

Figure 7 shows the cubic root of the M.W. of the luminophore as the axis $X$ and the ECL emission efficiency as the axis $Y$, where the M.W. of Ru-Ab, hIgG-Ru-Ab and Ru-chelate were 150000, 300000 and 750, respectively. This indicated remarkable inverse proportion between the cubic root of the M.W. of the luminophore and the ECL emission efficiency.

Generally, the diffusion speed of any molecule is inversely proportional to the diameter of the molecule. Since the electrolysis reaction occurs at only a limited distance from the surface of the electrode, the frequency of the electrolysis over the electrode might be inversely proportional to the molecular diameter, too. By using this phenomenon, the homogeneous ECLIA system might be more realistic if the molecular size of the luminophore could be significantly enlarged after the antigen-antibody reaction. For example, in the competitive ECLIA the luminophore might be labeled antigen with a smaller M.W. of less than 1000, or, as shown in Fig. 2b, in the sandwich type ECLIA conjunctive use of a solid phase carrier might be available for the large molecule. ${ }^{8,9}$

\section{Homogeneous sandwich ECLIA using Capt-MB and Ru-Ab}

Figure 8 shows the results of the 1-step sandwich homogeneous ECLIA obtained by LP-exc or by JM-exc, after the antigen-antibody reaction. In the case of LP-exc for the specimen reacted with $5 \mathrm{ng} \mathrm{Ru}-\mathrm{Ab}$ (3000 fold diluted solution), the ECL intensities were constantly at the 2000 a.u. level in the $\mathrm{hIgG}$ of 0 to $1 \mu \mathrm{g} / \mathrm{ml}$, and were gradually reduced to be 900 at the $\mathrm{hIgG}$ of $30 \mu \mathrm{g} / \mathrm{ml}$, which means that totally it reduced by 1100 a.u. In the case of increasing the $\mathrm{Ru}-\mathrm{Ab}$ amount up to 10 


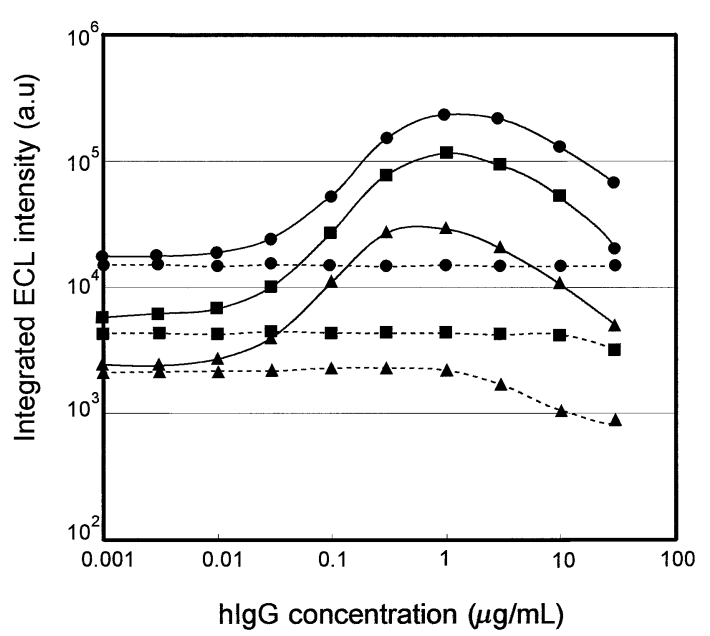

Fig. 8 Dose-response curves obtained by the 1-step sandwich homogeneous and LP-exc or JM-exc of ECL emissions. The dotted lines indicate the results of LP-exc and the solid lines indicate the results of JM-exc. The closed triangle ( $\mathbf{A}$ ) indicates the result of using $5 \mathrm{ng} \mathrm{Ru}-\mathrm{Ab}$, the closed square ( $\mathbf{\square})$ indicates that of $10 \mathrm{ng} \mathrm{Ru}-$ $\mathrm{Ab}$, and the closed circle ( $\bullet$ ) indicates that of $30 \mathrm{ng} \mathrm{Ru}-\mathrm{Ab}$.

ng (namely, a 1500 fold diluted solution), the base value of the ECL intensities increased to the 4000 level, which is about 2 times higher than that in the condition using $5 \mathrm{ng} \mathrm{Ru}-\mathrm{Ab}$. While in the hIgG concentration of 10 to $30 \mu \mathrm{g} / \mathrm{ml}$, the ECL intensities were reduced by 600 a.u. only, and not enough to deduce any dose-response curve. In the case of increasing the $\mathrm{Ru}-\mathrm{Ab}$ amount up to $30 \mathrm{ng}$ ( 300 fold diluted solution), the base value of the ECL intensities increased to more or less the 14000 level. There was not such a significant reduction in the ECL intensities as observed in the lower amount of Ru-Ab.

On the other hand, in the case of JM-exc for the reacted specimen by using any Ru-Ab amount, the ECL peaks appeared around $1 \mu \mathrm{g} / \mathrm{ml}$ of antigen concentration; the values of which were 14 to 20 times higher than the base values of all $\mathrm{Ru}-\mathrm{Ab}$ concentrations. Considering the relationship between the ECL intensities and the antigen concentrations, each ECL peak point seemed to be corresponding to the equivalent point of the antigen-antibody reaction. The clear dose-response curves appeared towards a lower antigen concentrations of 1 to 0.01 $\mu \mathrm{g} / \mathrm{ml}$, in the zone of antibody excess. Almost similar dose response curves but reverse correlated also appeared in the antigen excess zone. The ECL peak point formed by $10 \mathrm{ng} \mathrm{Ru}-$ $\mathrm{Ab}$ was the same as that by $30 \mathrm{ng} \mathrm{Ru}-\mathrm{Ab}$. This coincidence of ECL peak point may reflect that the binding capacity of the Capt-MB was saturated at a point of the $1 \mu \mathrm{g} / \mathrm{ml}$ of $\mathrm{hIgG}$ concentration. These results suggest that clearer dose response curves might be obtainable even from the LP-exc, with a higher quantity of Ru-Ab also, if a sufficient amount of Capt-MB is used for the antigen-antibody reaction concerned.

However, the above results definitely indicate that instead of pursuing the possibility of LP-exc at the zone of antigen excess, directly measuring the ECL signals for the specimen reacted with Capt-MB at the zone of antibody excess before reaching the equivalent point could be more reliable to obtain remarkably higher sensitivities and $\mathrm{S} / \mathrm{N}$ values.

\section{1-step and 2-step heterogeneous sandwich ECLIA}

Figures 9 and 10 show the results of the dose-response curves obtained by the 1-step and 2-step sandwich heterogeneous

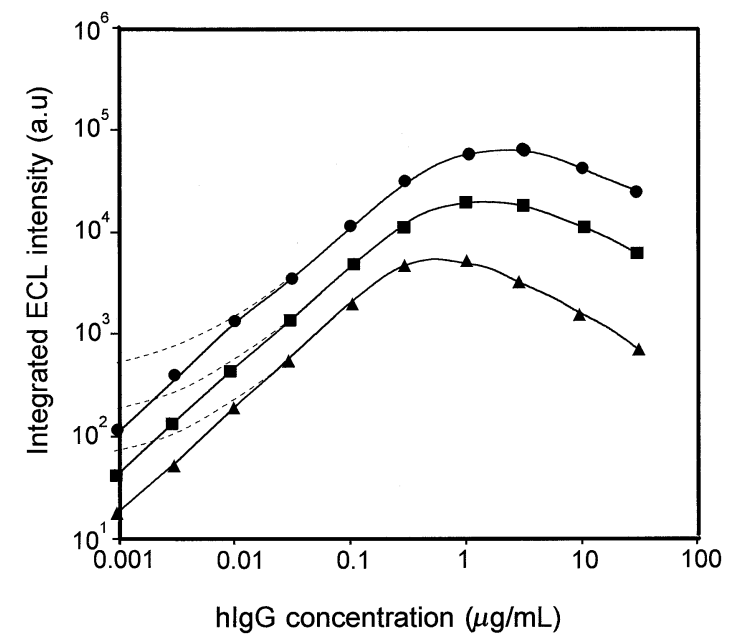

Fig. 9 Dose-response curves by the 1-step sandwich heterogeneous ECLIA by MT-exc. The solid lines indicate the results of the specific ECL intensities and the dotted lines include the blank value. The closed triangle ( $\Delta$ ) indicates the result of using $5 \mathrm{ng} \mathrm{Ru}-\mathrm{Ab}$, the closed square ( $\boldsymbol{\square})$ indicates that of $10 \mathrm{ng} \mathrm{Ru}-\mathrm{Ab}$, and the closed circle (•) indicates that of $30 \mathrm{ng} \mathrm{Ru}-\mathrm{Ab}$.

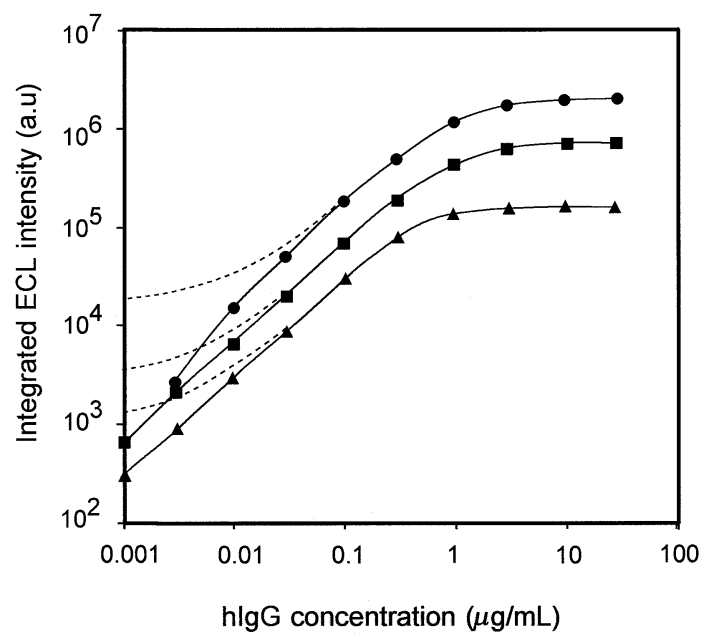

Fig. 10 Dose-response curves by the 2-step sandwich heterogeneous ECLIA by MT-exc. The solid lines indicate the results of the specific ECL intensities and the dotted lines include the blank value. The closed triangle ( $\Delta$ ) indicates the result of using $5 \mathrm{ng} \mathrm{Ru}-\mathrm{Ab}$, the closed square ( $\mathbf{a})$ indicates that of $10 \mathrm{ng} \mathrm{Ru}-\mathrm{Ab}$, and the closed circle (•) indicates that of $30 \mathrm{ng} \mathrm{Ru}-\mathrm{Ab}$.

ECLIA, respectively, involving both the $\mathrm{B} / \mathrm{F}$ separation and MT-exc. In the 1-step heterogeneous sandwich ECLIA, the dose-response curve of a specific ECL signal (minus blank value) was linear in the $\mathrm{hIgG}$ concentration range of $1 \mathrm{ng} / \mathrm{ml}$ to $300 \mathrm{ng} / \mathrm{ml}$, and the ECL signal also increased with the increase in $\mathrm{Ru}-\mathrm{Ab}$ amount. The hook phenomenon, caused by a shortage of the binding capacity of the Capt-MB and/or the Ru-Ab amount, was observed in the $\mathrm{hIgG}$ concentration range of 300 ng or more/ml in case of using the smallest $\mathrm{Ru}-\mathrm{Ab}$ amount of 5 ng. Likewise, the hook phenomena were also observed in the hIgG concentration range of $1 \mu \mathrm{g} / \mathrm{ml}$ to $3 \mu \mathrm{g} / \mathrm{ml}$ in the cases of using $10 \mathrm{ng}$ and $30 \mathrm{ng} \mathrm{Ru}-\mathrm{Ab}$. In corporation of the $\mathrm{B} / \mathrm{F}$ separation and MT-exc, the blank value was reduced to several tenth times that obtained by the homogeneous ECLIA with JM- 
exc (Figs. 8 and 9).

On the other hand, in the case of a 2-step sandwich heterogeneous ECLIA, where the F/B separations were incorporated before and after the reaction with the $\mathrm{Ru}-\mathrm{Ab}$, the ECL signals linearly reached the top point in the similar hook effect zone observed in the 1-step sandwich heterogeneous ECLIA. Moreover, no decrease in the ECL intensity was observed even after the antigen exceeded the equivalent point of antigen-antibody concentration. However, in the 2-step sandwich heterogeneous ECLIA, the ECL blanks caused by nonspecific adsorption of Ru-Ab were about 10 times as high as those in the 1-step ECLIA in every $\mathrm{Ru}-\mathrm{Ab}$ concentration. Consequently, in the case of using the $\mathrm{Ru}-\mathrm{Ab}$ of the highest amount of $30 \mathrm{ng}$, the sensitivity for the $100 \mathrm{ng} / \mathrm{ml}$ or below could not be obtained due to the high background.

It was considered that the reason why the nonspecific adsorption to the MB in the 2-step became higher than in the 1step might be ascribed to the lower protein concentration of the $\mathrm{Ru}-\mathrm{Ab}$ solution in the 2 nd reaction compared with the protein concentration in the 1-step assay which contains the more additional protein of the specimen component. Although this nonspecific adsorption may be reduced by an increase of the NRS concentration in the Ru-Ab solution, this difference in profile between 1-step and 2-step suggested that the 1-step assay system is suitable for obtaining higher sensitivity, while the 2-step assay system is suitable for obtaining a wider dynamic range.

To state our conclusion, our newly developed heterogeneous ECLIA made it possible to directly measure immune complexes bound with antibody-labeled luminophore before reaching the equivalent point of the antigen-antibody reaction. Accordingly, the $\mathrm{S} / \mathrm{N}$ ratio of the new ECLIA systems was improved by 1000 times or more over that of homogeneous ECLIA. Although the present report is not so much concerned with optimization of the assay conditions for obtaining high sensitivity, this study revealed the immunochemical and electrochemical background for the heterogeneous ECLIA to obtain high sensitivity, such as at the several picogram levels.

\section{References}

1. H. A. Rongen, R. M. Hoetelmans, A. Bult, and W. P. van Bennekom, J. Pharm. Biomed. Anal., 1994, 12, 433.

2. L. J. Kricka, Clin. Chem., 1991, 37, 1472.

3. Y. Namba, T. Naraki, K. Watanabe, S. Kida, Y. Iwasaki, T. Mukoushima, T. Kawai, R. J. Massey, and G. F. Blackburn, Jap. J. Cli. Phath, in Proceeding of 39th annual meeting, 1991, Asahikawa, Hokkaido, Japan, 56.

4. G. F. Blackburn, H. P. Shah, J. H. Kenten, J. K. Leland, R. A. Kamin, J. Link, J. Peterman, H. P. Shah, D. B. Talley, S. K. Tyagi, E. Wilkins, T. G. Wu, and R. J. Massey, Clin. Chem., 1991, 37, 1534.

5. A. Tsuji, and T. Sugano, "Chemiluminescence immunoassays", 1992, Life Science, Tokyo, Japan, 17.

6. A. J. Bard and L. R. Faulkner, "Electrochemical Methods: Fundamentals and Applications", 1980, John Wiley \& Sons, New York, 488.

7. Y. Ikariyama, H. Kunoh, and M. Aizawa, Biochem. Biophys. Res. Commun., 1985, 128, 987.

8. R. J. Massay, M. J. Powell, P. A. Mied, P. Feng, C. L. Della, W. J. Dressick, and M. S. Poonian, 1987, PCT WO 87/06706.

9. H. P. Shah, L. O. Hall, M. J. Powell, and R. J. Massey, 1990, PCT WO 90/05301.

10. J. K. Leland and M. Powell, J. Electrochem. Soc., 1990, 137, 3127.

11. Y. Namba, M. Usami, and O. Suzuki, Anal. Sci., 1999, 15 , 1087. 